\title{
Article \\ Semi-Hyers-Ulam-Rassias Stability of the Convection Partial Differential Equation via Laplace Transform
}

\author{
Daniela Marian (D)
}

check for

updates

Citation: Marian, D

Semi-Hyers-Ulam-Rassias Stability of the Convection Partial Differential Equation via Laplace Transform. Mathematics 2021, 9, 2980. https:/ / doi.org/10.3390/math9222980

Academic Editor: Janusz Brzdęk

Received: 23 October 2021

Accepted: 20 November 2021

Published: 22 November 2021

Publisher's Note: MDPI stays neutral with regard to jurisdictional claims in published maps and institutional affiliations.

Copyright: (c) 2021 by the authors. Licensee MDPI, Basel, Switzerland. This article is an open access article distributed under the terms and conditions of the Creative Commons Attribution (CC BY) license (https:/ / creativecommons.org/licenses/by/ $4.0 /)$.
Department of Mathematics, Technical University of Cluj-Napoca, 28 Memorandumului Street, 400114 Cluj-Napoca, Romania; daniela.marian@math.utcluj.ro,

Abstract: In this paper, we study the semi-Hyers-Ulam-Rassias stability and the generalized semiHyers-Ulam-Rassias stability of some partial differential equations using Laplace transform. One of them is the convection partial differential equation.

Keywords: semi-Hyers-Ulam-Rassias stability; generalized semi-Hyers-Ulam-Rassias stability; Laplace transform; convection partial differential equation

MSC: 44A10; 35B35

\section{Introduction}

It is well known that the study of Ulam stability began in 1940, with a problem posed by Ulam concerning the stability of homomorphisms [1]. In 1941, Hyers [2] gave a partial answer in the case of the additive Cauchy equation in Banach spaces.

After that, Obloza [3] and Alsina and Ger [4] began the study of the Hyers-Ulam stability of differential equations. The field continued to develop rapidly. Linear differential equations were studied in [5-7], integral equations in [8], delay differential equations in [9], linear difference equations in [10,11], other equations in [12], and systems of differential equations in [13]. A summary of these results can be found in [14].

The Hyers-Ulam stability of linear differential equations was studied using the Laplace transform by H. Rezaei, S. M. Jung, and Th. M. Rassias [15], and by Q. H. Alqifiary and S. M. Jung [16]. This method was also used in [17-19].

The study of the stability of partial differential equations began in 2003, with the paper [20] of A. Prastaro and Th.M. Rassias. The Ulam-Hyers stability of partial differential equations was also studied in [21-26].

In [27], M. N. Qarawani used the Laplace transform to establish the Hyers-Ulam-RassiasGavruta stability of initial-boundary value problem for heat equations on a finite rod:

$$
\frac{\partial u}{\partial t}=a^{2} \frac{\partial^{2} u}{\partial x^{2}}, t>0,0<x<l
$$

In [28], D.O. Deborah and A. Moyosola studied nonlinear, nonhomogeneous partial differential equations using the Laplace differential transform method:

$$
\frac{d^{2} w(x, t)}{d t^{2}}+a_{n}(x) \operatorname{R} w(x, t)+b_{n}(x) S w(x, t)=f(x, t), t>0, x>0, n \in \mathbb{N},
$$

where $a_{n}(x), b_{n}(x)$ are variable coefficients, $n \in \mathbb{N}, R$ is the linear operator, $S$ is the nonlinear operator, and $f(x, t)$ is the source function.

In [29], E. Bicer used the Sumudu transform to study the equation:

$$
y_{t}-k y_{x x}=0, k \text { a positive real constant, }(x, t) \in D, D=\left(x_{0}, x\right] \times(0, \infty) \text {. }
$$


In [30], the Poisson partial differential equation

$$
u_{x x}(x, y)+u_{y y}(x, y)=g(x, y)
$$

is studied via the double Laplace transform method (DLTM).

In the following sections, we will study the semi-Hyers-Ulam-Rassias stability and the generalized semi-Hyers-Ulam-Rassias stability of some partial differential equations using Laplace transform. One of them is the convection partial differential equation:

$$
\frac{\partial y}{\partial t}+a \frac{\partial y}{\partial x}=0, a>0, x>0, t>0, y(0, t)=c, y(x, 0)=0 .
$$

A physical interpretation [31] of these equations is a river of solid goo, since we do not want anything to diffuse. The function $y=y(x, t)$ is the concentration of some toxic substance. The variable $x$ denotes the position where $x=0$ is the location of a factory spewing the toxic substance into the river. The toxic substance flows into the river so that at $x=0$, the concentration is always $C$. We also study the semi-Hyers-Ulam-Rassias stability of the following equation:

$$
\frac{\partial y}{\partial t}+\frac{\partial y}{\partial x}-x=0, x>0, t>0, y(0, t)=0, y(x, 0)=0 .
$$

Our results regarding Equation (1) complete those obtained by S.-M. Jung and K.-S. Lee in [22]. In [22], the following equation:

$$
a \frac{\partial y(x, t)}{\partial x}+b \frac{\partial y(x, t)}{\partial t}+c y(x, t)+d=0, a, b \in \mathbb{R}, b \neq 0, c, d \in \mathbb{C}, \text { with } \Re(c) \neq 0,
$$

where $\Re(c)$ denotes the real part of $c$, was studied. In our paper, we consider the case $c=0$ in Equation (3). Moreover, we also study the generalized stability. The method used in [22] was the method of changing variables.

\section{Preliminaries}

We first recall some notions and results regarding the Laplace transform.

Let $f:(0, \infty) \rightarrow \mathbb{R}$ be a piecewise differentiable and of exponential order, that is $\exists M>0$ and $\alpha_{0} \geq 0$ such that

$$
|f(t)| \leq M \cdot e^{\alpha_{0} t}, \quad \forall t>0 .
$$

We denote by $\mathcal{L}[f]$ the Laplace transform of the function $f$, defined by

$$
\mathcal{L}[f](s)=F(s)=\int_{0}^{\infty} f(t) e^{-s t} d t .
$$

Let

$$
u(t)= \begin{cases}0, & \text { if } t \leq 0 \\ 1, & \text { if } t>0\end{cases}
$$

be the unit step function of Heaviside. We write $f(0)$ instead of the lateral limit $f\left(0^{+}\right)$. The following properties are used in the paper:

$$
\begin{gathered}
\mathcal{L}\left[t^{n}\right](s)=\frac{n !}{s^{n+1}}, s>0, n \in \mathbb{N}, \\
\mathcal{L}^{-1}\left[\frac{1}{s^{n}}\right](t)=\frac{t^{n-1}}{(n-1) !} u(t), \\
\mathcal{L}\left[f^{\prime}\right](s)=s \mathcal{L}[f](s)-f(0), \\
\mathcal{L}[f(t-a) u(t-a)](s)=e^{-a s} F(s), a>0,
\end{gathered}
$$


hence,

$$
\mathcal{L}^{-1}\left[e^{-a s} F(s)\right](t)=f(t-a) u(t-a) .
$$

We now consider the function $y:(0, \infty) \times(0, \infty) \rightarrow R, y=y(x, t)$, a piecewise differentiable and of exponential order with respect to $t$. The Laplace transform of $y$ with respect to $t$ is as follows:

$$
\mathcal{L}[y(x, t)]=\int_{0}^{\infty} y(x, t) e^{-s t} d t,
$$

where $x$ is treated as a constant. We also denote the following:

$$
\mathcal{L}[y(x, t)]=Y(x, s)=Y(x)=Y .
$$

We treat $Y$ as a function of $x$, leaving $s$ as a parameter. We then have the following:

$$
\begin{gathered}
\mathcal{L}\left[\frac{\partial y}{\partial t}\right]=s Y(x, s)-y(x, 0), \\
\mathcal{L}\left[\frac{\partial^{2} y}{\partial t^{2}}\right]=s^{2} Y(x, s)-s y(x, 0)-\frac{\partial y}{\partial t}(x, 0) .
\end{gathered}
$$

Since we transform with respect to $t$, we can move $\frac{\partial}{\partial x}$ to the front of the integral; hence, we have:

$$
\mathcal{L}\left[\frac{\partial y}{\partial x}\right]=\frac{d Y}{d x}=Y^{\prime}(x)
$$

Similarly,

$$
\mathcal{L}\left[\frac{\partial^{2} y}{\partial x^{2}}\right]=\int_{0}^{\infty} \frac{\partial^{2} y}{\partial x^{2}} e^{-s t} d t=\frac{d}{d x^{2}} \int_{0}^{\infty} y(x, t) e^{-s t} d t=\frac{d Y}{d x^{2}}=Y^{\prime \prime}(x) .
$$

For the Laplace transform properties and applications, see [31,32].

\section{Semi-Hyers-Ulam-Rassias Stability of the Convection Partial Differential Equation}

Let $\varepsilon>0$. We also consider the following inequality:

$$
\left|\frac{\partial y}{\partial t}+a \frac{\partial y}{\partial x}\right| \leq \varepsilon,
$$

or the equivalent

$$
-\varepsilon \leq \frac{\partial y}{\partial t}+a \frac{\partial y}{\partial x} \leq \varepsilon .
$$

Analogous to [33], we give the following definition:

Definition 1. The Equation (1) is called semi-Hyers-Ulam-Rassias stable if there exists a function $\varphi:(0, \infty) \times(0, \infty) \rightarrow(0, \infty)$, such that for each solution $y$ of the inequality (4), there exists a solution $y_{0}$ for the Equation (1) with

$$
\left|y(x, t)-y_{0}(x, t)\right| \leq \varphi(x, t), \quad \forall x>0, t>0 .
$$

Theorem 1. If a function $y:(0, \infty) \times(0, \infty) \rightarrow \mathbb{R}$ satisfies the inequality (4), then there exists a solution $y_{0}:(0, \infty) \times(0, \infty) \rightarrow \mathbb{R}$ for $(1)$, such that

$$
\left|y(x, t)-y_{0}(x, t)\right| \leq\left\{\begin{array}{l}
\varepsilon t, t<\frac{x}{a} \\
\varepsilon \frac{x}{a}, t \geq \frac{x}{a}
\end{array},\right.
$$

that is, the Equation (1) is considered semi-Ulam-Hyers-Rassias stable. 
Proof. We apply the Laplace transform with respect to $t$ in (5); thus, we have the following:

$$
-\frac{\varepsilon}{s} \leq s Y(x)-y(x, 0)+a Y^{\prime}(x) \leq \frac{\varepsilon}{s} .
$$

Since $y(x, 0)=0$, dividing by $a$ we get the following:

$$
-\frac{\varepsilon}{a s} \leq Y^{\prime}(x)+\frac{s}{a} Y(x) \leq \frac{\varepsilon}{a s} .
$$

We now multiply by $e^{\frac{s}{a} x}$ and we obtain this equation:

$$
-\frac{\varepsilon}{a s} e^{\frac{s}{a} x} \leq e^{\frac{s}{a} x} Y^{\prime}(x)+\frac{s}{a} e^{\frac{s}{a} x} Y(x) \leq \frac{\varepsilon}{a s} e^{\frac{s}{a} x},
$$

hence,

$$
-\frac{\varepsilon}{a s} e^{\frac{s}{a} x} \leq \frac{d}{d x}\left(e^{\frac{s}{a} x} Y(x)\right) \leq \frac{\varepsilon}{a s} e^{\frac{s}{a} x} .
$$

Integrating from 0 to $x$ we get the following:

$$
-\left.\frac{\varepsilon}{a s} \frac{e^{\frac{s}{a} x}}{\frac{s}{a}}\right|_{0} ^{x}<\left.e^{\frac{s}{a} x} Y(x)\right|_{0} ^{x} \leq\left.\frac{\varepsilon}{a s} e^{\frac{s}{a} x}\right|_{0} ^{x}
$$

that is,

$$
-\varepsilon\left(\frac{e^{\frac{s}{a} x}}{s^{2}}-\frac{1}{s^{2}}\right) \leq e^{\frac{s}{a} x} Y(x)-Y(0) \leq \varepsilon\left(\frac{e^{\frac{s}{a} x}}{s^{2}}-\frac{1}{s^{2}}\right) .
$$

But $Y(0)=\mathcal{L}[y(0, t)]=\mathcal{L}[c]=\frac{c}{s}$, so we obtain:

$$
-\varepsilon\left(\frac{e^{\frac{s}{a} x}}{s^{2}}-\frac{1}{s^{2}}\right) \leq e^{\frac{s}{a} x} Y(x)-\frac{c}{s} \leq \varepsilon\left(\frac{e^{\frac{s}{a} x}}{s^{2}}-\frac{1}{s^{2}}\right) .
$$

We now multiply by $e^{-\frac{s}{a} x}$ and we obtain the equation below:

$$
-\varepsilon\left(\frac{1}{s^{2}}-\frac{e^{-\frac{s}{a} x}}{s^{2}}\right) \leq Y(x)-c \frac{e^{-\frac{s}{a} x}}{s} \leq \varepsilon\left(\frac{1}{s^{2}}-\frac{e^{-\frac{s}{a} x}}{s^{2}}\right) .
$$

We apply the inverse Laplace transform and we obtain the following:

$$
-\varepsilon\left[t-\left(t-\frac{x}{a}\right) u\left(t-\frac{x}{a}\right)\right] \leq y(x, t)-c \cdot u\left(t-\frac{x}{a}\right) \leq \varepsilon\left[t-\left(t-\frac{x}{a}\right) u\left(t-\frac{x}{a}\right)\right],
$$

that is,

$$
\left|y(x, t)-c \cdot u\left(t-\frac{x}{a}\right)\right| \leq \varepsilon\left[t-\left(t-\frac{x}{a}\right) u\left(t-\frac{x}{a}\right)\right] .
$$

We then put

$$
y_{0}(x, t)=c \cdot u\left(t-\frac{x}{a}\right)=\left\{\begin{array}{l}
0, t<\frac{x}{a} \\
c, t \geq \frac{x}{a}
\end{array} .\right.
$$

This is the solution of (1) and the equation below:

$$
\left|y(x, t)-y_{0}(x, t)\right| \leq\left\{\begin{array}{l}
\varepsilon t, t<\frac{x}{a} \\
\varepsilon \frac{x}{a}, t \geq \frac{x}{a}
\end{array} .\right.
$$




\section{Generalized Semi-Hyers-Ulam-Rassias Stability of the Convection Partial Differential Equation}

Let $\phi:(0, \infty) \times \mathbb{R} \rightarrow(0, \infty)$, and $\mathcal{L}[\phi(x, t)]=\Phi(x, s)$. We consider the following inequality:

$$
\left|\frac{\partial y}{\partial t}+a \frac{\partial y}{\partial x}\right| \leq \phi(x, t)
$$

or the equivalent

$$
-\phi(x, t) \leq \frac{\partial y}{\partial t}+a \frac{\partial y}{\partial x} \leq \phi(x, t), \quad \forall x>0, t>0 .
$$

Definition 2. The Equation (1) is called generalized semi-Hyers-Ulam-Rassias stable if there exists a function $\varphi:(0, \infty) \times(0, \infty) \rightarrow(0, \infty)$, such that for each solution $y$ of the inequality $(7)$, there exists a solution $y_{0}$ for the Equation (1) with

$$
\left|y(x, t)-y_{0}(x, t)\right| \leq \varphi(x, t), \quad \forall x>0, t>0 .
$$

Theorem 2. Assume that

$$
\int_{0}^{x} e^{\frac{s}{a} x} \Phi(x, s) d x \leq \Phi(x, s), \quad \forall x>0, s>0 .
$$

If a function $y:(0, \infty) \times(0, \infty) \rightarrow \mathbb{R}$ satisfies the inequality (7), then there exists a solution $y_{0}:(0, \infty) \times(0, \infty) \rightarrow \mathbb{R}$ for $(1)$, such that

$$
\left|y(x, t)-y_{0}(x, t)\right| \leq \frac{1}{a} \phi\left(x, t-\frac{x}{a}\right), \quad \forall x>0, t>0,
$$

that is, the Equation (1) is considered generalized semi-Hyers-Ulam-Rassias stable.

Proof. We apply the Laplace transform with respect to $t$ in (8), so we have the following:

$$
-\Phi(x, s) \leq s Y(x)-y(x, 0)+a Y^{\prime}(x) \leq \Phi(x, s) .
$$

Since $y(x, 0)=0$, dividing by $a$ we get the equation below:

$$
-\frac{1}{a} \Phi(x, s) \leq Y^{\prime}(x)+\frac{s}{a} Y(x) \leq \frac{1}{a} \Phi(x, s) .
$$

We now multiply by $e^{\frac{s}{a} x}$ and we obtain the following:

$$
-\frac{e^{\frac{s}{a} x}}{a} \Phi(x, s) \leq e^{\frac{s}{a} x} Y^{\prime}(x)+\frac{s}{a} e^{\frac{s}{a} x} Y(x) \leq \frac{e^{\frac{s}{a} x}}{a} \Phi(x, s),
$$

hence,

$$
-\frac{e^{\frac{s}{a} x}}{a} \Phi(x, s) \leq \frac{d}{d x}\left(e^{\frac{s}{a} x} Y(x)\right) \leq \frac{e^{\frac{s}{a} x}}{a} \Phi(x, s) .
$$

Integrating from 0 to $x$ we get the following equation:

$$
-\frac{1}{a} \int_{0}^{x} e^{\frac{s}{a} x} \Phi(x, s) d x \leq\left. e^{\frac{s}{a} x} Y(x)\right|_{0} ^{x} \leq \int_{0}^{x} \frac{1}{a} e^{\frac{s}{a} x} \Phi(x, s) d x .
$$

Using (9), we have

$$
-\frac{1}{a} \Phi(x, s) \leq e^{\frac{s}{a} x} Y(x)-Y(0) \leq \frac{1}{a} \Phi(x, s) .
$$


But $Y(0)=L[y(0, t)]=L[c]=\frac{c}{s}$, so we obtain

$$
-\frac{1}{a} \Phi(x, s) \leq e^{\frac{s}{a} x} Y(x)-\frac{c}{s} \leq \frac{1}{a} \Phi(x, s) .
$$

We now multiply by $e^{-\frac{s}{a} x}$ and we obtain the following equation:

$$
-\frac{1}{a} e^{-\frac{s}{a} x} \Phi(x, s) \leq Y(x)-c \frac{e^{-\frac{s}{a} x}}{s} \leq \frac{1}{a} e^{-\frac{s}{a} x} \Phi(x, s) .
$$

We apply the inverse Laplace transform and we obtain:

$$
-\frac{1}{a} \phi\left(x, t-\frac{x}{a}\right) \leq y(x, t)-c \cdot u\left(t-\frac{x}{a}\right) \leq \frac{1}{a} \phi\left(x, t-\frac{x}{a}\right),
$$

that is,

$$
\left|y(x, t)-c \cdot u\left(t-\frac{x}{a}\right)\right| \leq \frac{1}{a} \phi\left(x, t-\frac{x}{a}\right) .
$$

We then put the following:

$$
y_{0}(x, t)=c \cdot u\left(t-\frac{x}{a}\right)=\left\{\begin{array}{l}
0, t<\frac{x}{a} \\
c, t \geq \frac{x}{a}
\end{array} .\right.
$$

This is the solution of Equation (1) and the equation below:

$$
\left|y(x, t)-c y_{0}(x, t)\right| \leq \frac{1}{a} \phi\left(x, t-\frac{x}{a}\right) .
$$

\section{Semi-Hyers-Ulam-Rassias Stability of Equation (2)}

Let $\varepsilon>0$. We also consider the following inequality:

$$
\left|\frac{\partial y}{\partial t}+\frac{\partial y}{\partial x}-x\right| \leq \varepsilon,
$$

or the equivalent

$$
-\varepsilon \leq \frac{\partial y}{\partial t}+\frac{\partial y}{\partial x}-x \leq \varepsilon
$$

Definition 3. The Equation (2) is called semi-Hyers-Ulam-Rassias stable if there exists a function $\varphi:(0, \infty) \times(0, \infty) \rightarrow(0, \infty)$, such that for each solution $y$ of the inequality $(10)$, there exists a solution $y_{0}$ for the Equation (2) with the following:

$$
\left|y(x, t)-y_{0}(x, t)\right| \leq \varphi(x, t), \quad \forall x>0, t>0 .
$$

Theorem 3. If a function $y:(0, \infty) \times(0, \infty) \rightarrow \mathbb{R}$ satisfies the inequality (10), then there exists a solution $y_{0}:(0, \infty) \times(0, \infty) \rightarrow \mathbb{R}$ for $(2)$, such that

$$
\left|y(x, t)-y_{0}(x, t)\right| \leq\left\{\begin{array}{l}
\varepsilon t, t<x \\
\varepsilon x, t \geq x
\end{array}\right.
$$

that is, the Equation (2) is considered semi-Hyers-Ulam-Rassias stable.

Proof. We apply the Laplace transform with respect to $t$ in (11), so we have the equation below:

$$
-\frac{\varepsilon}{S} \leq s Y(x)-y(x, 0)+Y^{\prime}(x)-x \frac{1}{s} \leq \frac{\varepsilon}{S} .
$$


Since $y(x, 0)=0$, we get the following:

$$
-\frac{\varepsilon}{s} \leq Y^{\prime}(x)+s Y(x)-x \frac{1}{s} \leq \frac{\varepsilon}{s} .
$$

We now multiply by $e^{s x}$ and we obtain the following equation:

$$
-\frac{\varepsilon}{s} e^{s x} \leq e^{s x} Y^{\prime}(x)+s e^{s x} Y(x)-x \frac{e^{s x}}{s} \leq \frac{\varepsilon}{s} e^{s x} .
$$

hence,

$$
-\frac{\varepsilon}{s} e^{s x} \leq \frac{d}{d x}\left(e^{s x} Y(x)\right)-x \frac{e^{s x}}{s} \leq \frac{\varepsilon}{s} e^{s x} .
$$

Integrating from 0 to $x$, we get the following:

$$
-\left.\frac{\varepsilon}{s} \frac{e^{s x}}{s}\right|_{0} ^{x} \leq\left. e^{s x} Y(x)\right|_{0} ^{x}-\frac{1}{s} \int_{0}^{x} x e^{s x} d x \leq\left.\frac{\varepsilon}{s} \frac{e^{s x}}{s}\right|_{0} ^{x} .
$$

Integrating by parts, we get the equation below:

$$
\int_{0}^{x} x e^{s x} d x=\frac{(x s-1) e^{s x}}{s^{2}}+\frac{1}{s^{2}}
$$

hence,

$$
-\varepsilon\left(\frac{e^{s x}}{s^{2}}-\frac{1}{s^{2}}\right) \leq e^{s x} Y(x)-Y(0)-\frac{1}{s}\left[\frac{(x s-1) e^{s x}}{s^{2}}+\frac{1}{s^{2}}\right] \leq \varepsilon\left(\frac{e^{s x}}{s^{2}}-\frac{1}{s^{2}}\right) .
$$

But $Y(0)=\mathcal{L}[y(0, t)]=0$, so we obtain the following:

$$
-\varepsilon\left(\frac{e^{s x}}{s^{2}}-\frac{1}{s^{2}}\right) \leq e^{s x} Y(x)-\frac{1}{s}\left[\frac{(x s-1) e^{s x}}{s^{2}}+\frac{1}{s^{2}}\right] \leq \varepsilon\left(\frac{e^{s x}}{s^{2}}-\frac{1}{s^{2}}\right) .
$$

We now multiply by $e^{-s x}$ and we obtain the following:

$$
-\varepsilon\left(\frac{1}{s^{2}}-\frac{e^{-s x}}{s^{2}}\right) \leq Y(x)-\frac{1}{s}\left[\frac{x s-1}{s^{2}}+\frac{e^{-s x}}{s^{2}}\right] \leq \varepsilon\left(\frac{1}{s^{2}}-\frac{e^{-s x}}{s^{2}}\right),
$$

hence,

$$
-\varepsilon\left(\frac{1}{s^{2}}-\frac{e^{-s x}}{s^{2}}\right) \leq Y(x)-\frac{x}{s^{2}}+\frac{1}{s^{3}}-\frac{e^{-s x}}{s^{3}} \leq \varepsilon\left(\frac{1}{s^{2}}-\frac{e^{-s x}}{s^{2}}\right) .
$$

We apply the inverse Laplace transform and we obtain the following equation:

$$
-\varepsilon[t-(t-x) u(t-x)] \leq y(x, t)-x t+\frac{1}{2} t^{2}-\frac{1}{2}(t-x)^{2} u(t-x) \leq \varepsilon[t-(t-x) u(t-x)] .
$$

We then put the following:

$$
y_{0}(x, t)=x t-\frac{1}{2} t^{2}+\frac{1}{2}(t-x)^{2} u(t-x)=\left\{\begin{array}{l}
x t-\frac{1}{2} t^{2}, t<x \\
\frac{1}{2} x^{2}, t \geq x
\end{array} .\right.
$$

This is the solution of (2) and the equation below:

$$
\left|y(x, t)-y_{0}(x, t)\right| \leq\left\{\begin{array}{l}
\varepsilon t, t<x \\
\varepsilon x, t \geq x
\end{array} .\right.
$$




\section{Conclusions}

In this paper, we studied the semi-Hyers-Ulam-Rassias stability of Equations (1) and (2) and the generalized semi-Hyers-Ulam-Rassias stability of Equation (1) using the Laplace transform. To the best of our knowledge, the Hyers-Ulam-Rassias stability of Equations (1) and (2) has not been discussed in the literature with the use of the Laplace transform method. Our results complete those of Jung and Lee [22]. In [22], the Equation (3) was studied for $\Re(c) \neq 0$. We considered the case $c=0$ in Equation (3). We can apply our results to the convection equation in the sense that for every solution $y$ of (4), which is called an approximate solution, there exists an exact solution $y_{0}$ of (1), such that the relation (6) is satisfied. From a different perspective, the approximate solution can be viewed in relation to the perturbation theory, as any approximate solution of (4) is an exact solution of the perturbed equation $\frac{\partial y}{\partial t}+a \frac{\partial y}{\partial x}=h(x, t),|h(x, t)| \leq \varepsilon, a>0, x>0$, $t>0, y(0, t)=c, y(x, 0)=0$.

We intend to study other partial differential equations as well as other integro-differential equations using this method. We have already applied this method to [34], where we investigated the semi-Hyers-Ulam-Rassias stability of a Volterra integro-differential equation of order I with a convolution-type kernel.

Funding: This research received no external funding.

Institutional Review Board Statement: Not applicable.

Informed Consent Statement: Not applicable.

Conflicts of Interest: The author declares no conflict of interest.

\section{References}

1. Ulam, S.M. A Collection of Mathematical Problems; Interscience: New York, NY, USA, 1960.

2. Hyers, D.H. On the stability of the linear functional equation. Proc. Natl. Acad. Sci. USA 1941, 27, 222-224. [CrossRef]

3. Obloza, M. Hyers stability of the linear differential equation. Rocznik Nauk-Dydakt. Prace Mat. 1993, 13, 259-270.

4. Alsina, C.; Ger, R. On some inequalities and stability results related to exponential function. J. Inequal. Appl. 1998, 2, 373-380. [CrossRef]

5. Cimpean, D.S.; Popa, D. On the stability of the linear differential equation of higher order with constant coefficients. Appl. Math. Comput. 2010, 217, 4141-4146. [CrossRef]

6. Jung, S.-M. Hyers-Ulam stability of linear differential equations of first order, III. J. Math. Anal. Appl. 2005, 311, 139-146. [CrossRef]

7. Takahasi, S.E.; Takagi, H.; Miura, T.; Miyajima, S. The Hyers-Ulam stability constant of first order linear differential operators. J. Math. Anal. Appl. 2004, 296, 403-409. [CrossRef]

8. Marian, D.; Ciplea, S.A.; Lungu, N. On a functional integral equation. Symmetry 2021, 13, 1321. [CrossRef]

9. Otrocol, D.; Ilea, V.A. Ulam stability for a delay differential equation. Cent. Eur. J. Math. 2013, 11, 1296-1303. [CrossRef]

10. Baias, A.R.; Popa, D. On Ulam stability of a linear difference equation in Banach spaces. Bull. Malaysian Math. Sci. Soc. 2020, 43, 1357-1371. [CrossRef]

11. Novac, A.; Otrocol, D.; Popa, D. Ulam stability of a linear difference equation in locally convex spaces. Results Math. 2021, 76, 1-13. [CrossRef]

12. Otrocol, D. Ulam stabilities of differential equation with abstract Volterra operator in a Banach space. Nonlinear Funct. Anal. Appl. 2010, 15, 613-619.

13. Marian, D.; Ciplea, S.A.; Lungu, N. On Ulam-Hyers stability for a system of partial differential equations of first order. Symmetry 2020, 12, 1060. [CrossRef]

14. Brzdek, J.; Popa, D.; Rasa, I.; Xu, B. Ulam Stability of Operators; Elsevier: Amsterdam, The Netherlands, 2018.

15. Rezaei, H.; Jung, S.-M.; Rassias, T. Laplace transform and Hyers-Ulam stability of linear differential equations. J. Math. Anal. Appl. 2013, 403, 244-251. [CrossRef]

16. Alqifiary, Q.; Jung, S.-M. Laplace transform and generalized Hyers-Ulam stability of linear differential equations. Electron. J. Differ. Equ. 2014, 2014, 1-11.

17. Bicer, E.; Tunc, C. On the Hyers-Ulam Stability of Laguerre and Bessel Equations by Laplace Transform Method. Nonlinear Dyn. Syst. 2017, 17, 340-346.

18. Murali, R.; Ponmana Selvan, A. Mittag-Leffler-Hyers-Ulam stability of a linear differential equation of first order using Laplace transforms. Canad. J. Appl. Math. 2020, 2, 47-59.

19. Shen, Y.; Chen, W. Laplace Transform Method for the Ulam Stability of Linear Fractional Differential Equations with Constant Coefficients. Mediterr. J. Math. 2017, 14, 25. [CrossRef] 
20. Prastaro, A.; Rassias, T.M. Ulam stability in geometry of PDE's. Nonlinear Funct. Anal. Appl. 2003, 8, $259-278$.

21. Jung, S.-M. Hyers-Ulam stability of linear partial differential equations of first order. Appl. Math. Lett. 2009, 22, 70-74. [CrossRef]

22. Jung, S.-M.; Lee, K.-S. Hyers-Ulam stability of first order linear partial differential equations with constant coefficients. Math. Inequal. Appl. 2007, 10, 261-266. [CrossRef]

23. Lungu, N.; Ciplea, S. Ulam-Hyers-Rassias stability of pseudoparabolic partial differential equations. Carpatian J. Math. 2015, 31, 233-240. [CrossRef]

24. Lungu, N.; Marian, D. Ulam-Hyers-Rassias stability of some quasilinear partial differential equations of first order. Carpathian J. Math. 2019, 35, 165-170. [CrossRef]

25. Lungu, N.; Popa, D. Hyers-Ulam stability of a first order partial differential equation. J. Math. Anal. Appl. 2012, 385, 86-91. [CrossRef]

26. Marian, D.; Ciplea, S.A.; Lungu, N. Ulam-Hyers stability of Darboux-Ionescu problem. Carpatian J. Math. 2021, 37, 211-216. [CrossRef]

27. Qarawani, M.N. Hyers-Ulam-Rassias Stability for the Heat Equation. Appl. Math. 2013, 4, 1001-1008. [CrossRef]

28. Deborah, D.O.; Moyosola, A. Laplace differential transform method for solving nonlinear nonhomogeneous partial differential equations. Turk. J. Anal. Number Theory 2020, 8, 91-96. [CrossRef]

29. Bicer, E. Applications of Sumudu transform method for Hyers-Ulam stability of partial differential equations. J. Appl. Math. Inform. 2021, 39, 267-275.

30. Abdulah, A.A.; Ahmad, A. The solution of Poisson partial differential equations via double Laplace transform method. Partial Differ. Equ. Appl. Math. 2021, 4, 100058.

31. Lebl, J. Notes on Diffy Qs: Differential Equations for Engineers; CreateSpace Independent Publishing Platform: Charleston, SC, USA, 2021.

32. Cohen, A.M. Numerical Methods for Laplace Transform Inversion (Numerical Methods and Algorithms, 5); Springer: Berlin/Heidelberg, Germany, 2007.

33. Castro, L.P.; Simões, A.M. Different Types of Hyers-Ulam-Rassias Stabilities for a Class of Integro-Differential Equations. Filomat 2017, 31, 5379-5390. [CrossRef]

34. Inoan, D.; Marian, D. Semi-Hyers-Ulam-Rassias stability of a Volterra integro-differential equation of order I with a convolution type kernel via Laplace transform. Symmetry 2021, 13, 2181. [CrossRef] 